\title{
Importance of concrete material characterization and modelling to predicting the response of structures to shock and impact loading
}

\author{
J. M. Magallanes \\ Karagozian \& Case, Burbank, CA, USA
}

\begin{abstract}
This paper reviews the importance of concrete material characterization and modelling to predicting the response of reinforced concrete structures to impulsive loads using physics-based finite element models. Comparisons of several widely available concrete constitutive models are presented pertaining to their ability to reproduce basic laboratory data for concrete as well as predict the response of structures subjected to shock and impact loadings. The examples presented here illustrate important differences between the various models in addition to illustrating some key concrete behaviours that the models should be able to capture.
\end{abstract}

Keywords: concrete, material characterization, constitutive models, finite element, shock, blast, impact.

\section{Introduction}

Structural response predictions using physics-based Lagrangian finite element (FE) models with explicit time-integration are widely used by both academics and practitioners to determine the behaviours of reinforced concrete (RC) structures subjected to a variety of shock and impact loadings. As full-scale structural response test data is costly and difficult to obtain, verified and validated FE models afford the ability to generate consistent sets of virtual response data from which to study structural behaviours, formulate simplified engineering models, and develop new structural and material designs aimed at increasing resistance to these types of loads. The advancement of FE methods 
and material constitutive models has reached a point where these tools are readily employed by practitioners in support of everyday structural designs and assessments.

Unfortunately, attention to constitutive modelling, especially for highly damaged material states, is too often left underappreciated and material models are commonly used to simulate material behaviours outside their domain of applicability. Moreover, FE analysts are often faced with selecting from an array of different concrete constitutive models but may only have limited experience or background upon which to select the most appropriate model and then reliably deploy it.

The primary purpose of this paper is to present a systematic review of concrete material characterization, within the context of predicting the response of RC structures to impulsive loadings using physics-based FE models. This is done by first comparing several available concrete constitutive models with regards to their ability to reproduce basic laboratory material characterization data. This is done to illustrate the important differences between the models in addition to demonstrating the key concrete behaviours the models should be capable of reproducing. Then, these concrete models are applied to common structural response problems to assess the effect of the constitutive models on the calculated response.

\section{Constitutive models}

Four widely available concrete material constitutive models were selected for review, which are all available in the LS-DYNA code [1]. The HolmquistJohnson-Cook model (HJC) is a model originally developed for penetration/perforation physics [2]. This model features confinement and strainrate effects. The continuous surface cap model (CSC) is a model originally developed as a general geo-mechanical model [3]. Features of this model include confinement effects, a three-invariant failure surface, strain-rate effects, and brittle and/or ductile damage. The Karagozian and Case concrete model (KCC) is a model developed specifically for RC structural response to blast and impact loadings [4]. This model also provides confinement effects, a threeinvariant failure surface, strain-rate effects, and brittle and/or ductile damage. The brittle damage concrete (BDC) model developed by Govindjee et al [5] to model damage of brittle solids like concrete was also examined in this paper. Besides the KCC model, predictions for blast and impact problems using these and other constitutive models have been published with little evidence of their applicability to this class of problems.

Generally speaking, the primary difference between each of these constitutive models lies in the manner in which the deviatoric and volumetric responses are characterized. The latter is typically governed by an equation of state (EOS), while the former is governed by a yield function, $f$, of the form:

$$
f\left(J_{1}, J_{2}^{\prime}, J_{3}^{\prime}\right)=\sigma_{e}\left(J_{2}^{\prime}\right)-F\left(J_{1}, J_{3}^{\prime}\right)
$$

In eqn (1), $J_{I}$ is the first stress invariant (related to pressure, $p=J_{I} / 3$ ), $J_{2}$ is the second invariant of the deviatoric stress tensor and $J_{3}$ is the third invariant of the 
deviatoric stress tensor. Either the von Mises stress, $\sigma_{e}$, or $J_{2}$ ' are used in eqn (1), depending on the preferred numerical implementation of each model (recall that $\left.\sigma_{e}=\sqrt{3 \cdot J_{2}^{\prime}}\right) . F$ is the equation of the failure surface unique to each model.

\subsection{Holmquist-Johnson-Cook (HJC) concrete model}

The HJC model uses a failure surface based on two-invariants:

$$
F(p, D)=\left[A \cdot(1-D)+B \cdot\left(p / f_{c}^{\prime}\right)^{N}\right] \cdot\left[1+C \cdot \ln \underline{\varepsilon^{*}}\right] \cdot f_{c}^{\prime}
$$

In eqn. (2), $p$ is the pressure, $\underline{\varepsilon}^{*}$ is a dimensionless measure of deviatoric strain rate, and $A, B, C$, and $N$ are experimentally calibrated constants. $D$ is a damage parameter ranging from 0.0 to 1.0 and is a function of equivalent deviatoric and volumetric plastic strain.

The HJC uses an equation of state (EOS) that is a quadratic function of a modified volumetric strain $\mu$ that has three experimentally derived constants $\left(K_{1}\right.$, $K_{2}$, and $\left.K_{3}\right)$ :

$$
p(\bar{\mu})=K_{1} \cdot \bar{\mu}+K_{2} \cdot \bar{\mu}^{2}+K_{3} \cdot \bar{\mu}^{3}
$$

\subsection{Continuous Surface Cap (CSC) model}

The CSC model uses a more complex multiplicative and three-invariant form of a failure surface originally proposed by Sandler et al. [6]:

$$
\begin{gathered}
F\left(J_{1}, J_{3}, k\right)=F_{f}^{2} \cdot F_{c} \cdot R\left(J_{3}^{\prime}\right)^{2} \\
F_{f}\left(J_{1}\right)=\alpha-\lambda \cdot \exp \left(-\beta \cdot J_{1}\right)+\theta \cdot J_{1} \\
F_{c}\left(J_{1}, k\right)=\frac{\left[J_{1}-L(k)\right] \cdot\left[J_{1}-L(k) \mid+J_{1}-L(k)\right]}{2 \cdot[X(k)-L(k)]^{2}}
\end{gathered}
$$

In eqn (4), $F_{f}$ is the failure surface envelope that is defined with experimentally calibrated parameters $\alpha, \lambda, \beta$, and $\theta$ (eqn (5)); $F_{c}$ is an elliptical cap function (eqn (6)); and $R$ is a function that modifies $F$ as a function of $J_{3}$ according to the methodology proposed by Rubin [7]. The cap parameter, $k$, controls the movement of the cap surface (eqn (6)), which controls the volumetric behaviour of the material and provides coupling of the concrete's deviatoric and compaction responses. In eqn (6), $X(k)$ is the intersection of the cap with the $J_{l}$ axis (in $\sigma_{e}-J_{l}$ space), and $L(k)$ is the position of the axis where the cap intersects the shear failure surface $F_{f}$. Volumetric plastic strains, $e_{v}^{p}$, are accumulated according to the following relationship:

$$
e_{v}^{p}=W\left(1-\exp \left[-D_{1}\left(X(k)-X\left(k_{o}\right)-D_{2}\left(X(k)-X\left(k_{0}\right)^{2}\right]\right)\right.\right.
$$

In eqn (7), $W, D_{1}, D_{2}$, and $X\left(k_{0}\right)$ are all experimentally calibrated to hydrostatic compression test results. 


\subsection{Karagozian and Case Concrete (KCC) model}

The KCC model is a three-invariant model where the failure surface is derived by interpolating between two of three independent surfaces $\left(F_{y}, F_{m}, F_{r}\right)$ according to the following relationship:

$$
\begin{aligned}
& F\left(J_{1}, J_{3}^{\prime}, \eta\right)=\left[\eta(\lambda) \cdot\left(F_{m}-F_{y}\right)+F_{y}\right] \cdot r^{\prime}\left(J_{3}^{\prime}\right) \text { for } 0.0 \leq \lambda \leq 1.0 \\
& F\left(J_{1}, J_{3}^{\prime}, \eta\right)=\left[\eta(\lambda) \cdot\left(F_{m}-F_{r}\right)+F_{r}\right] \cdot r^{\prime}\left(J_{3}^{\prime}\right) \text { for } 1.0>\lambda \leq 2.0
\end{aligned}
$$

The surfaces employed represent: a surface $F_{y}$ indicating where damage begins, a surface $F_{m}$ representing the maximum strength of the material, and a surface $F_{r}$ representing the residual strength of the material (i.e., after the damage has destroyed the materials cohesion). As indicated in eqn (8), the value of $F$ depends on a damage function $\eta(\lambda)$, which is defined from experimental data. The failure surface is a function of $J_{3}$ ' via the function, $r$, an implementation of the William and Warnke equation [8]. The three independent surfaces, denoted here as $F_{i}$, each use a parabolic form defined with experimentally calibrated constants, $a_{0 i}, a_{1 i}$, and $a_{2 i}$ (9 parameters total for the three surfaces) as follows:

$$
F_{i}(p)=a_{0 i}+\frac{p}{a_{1 i}+a_{2 i} \cdot p}
$$

The KCC model was developed with the intent of being used in conjunction with any EOS model that could capture volumetric hardening. In contrast to the CSC model, the KCC model cannot produce compaction softening.

\subsection{Brittle Damage Concrete (BDC) model}

The BDC model provides a basic tensile softening capability for characterizing a concrete's behaviour. This is also a phenomenon captured by the CSC and KCC models, although this is not described in detail here for the sake of brevity. With respect to the failure surface, the BDC uses a one-invariant formulation for $f$, and thus, $F$ is taken as a scalar in this model.

\section{Comparison of analytic results with test data}

\subsection{Comparison of models under various stress paths}

The performance of each material model was first assessed by subjecting a single element (i.e., a single point integration) to various basic stress paths. These calculations were performed using LS-DYNA and the results were then compared with a recent set of laboratory data for concrete [9]. The parameters used to characterize each model were taken directly from the literature without any modification. Consequently, the individual constitutive fits are representative of a 40.7 $\mathrm{MPa}$ concrete for the $\mathrm{HJC}$ [10] and a $40.0 \mathrm{MPa}$ concrete for the CSC [1], KCC [11], and BDC [5] models, respectively. The laboratory data had an average unconfined compressive strength of $33.6 \mathrm{MPa}$ [9]. This material is referred to as the SAM-35 concrete. Because of this difference in 
strength (40 $\mathrm{MPa}$ versus $35 \mathrm{MPa}$ ), the subsequent discussions are focused on phenomenological rather than quantitative comparisons.

\subsubsection{Basic stress paths}

Figure 1 shows comparative results basic laboratory stress path tests, including unconfined compression, hydrostatic compression, and unconfined tension (sometimes referred to as direct tension). The peak axial stress measured in the unconfined compression test is the concrete's unconfined compressive strength, $f_{c}^{\prime}$. The HJC, CSC, and KCC models exhibit the classic hardening and softening behaviours exhibited by the SAM-35 test data under this stress path, while the BDC model, on the other hand, remains perfectly plastic after reaching its peak strength. This is a consequence of its single invariant formulation. Note also that the HJC model transitions via linear trajectories from the yield point, to $f_{c}^{\prime}$, and into softening regime. This is a limitation of the simple damage function employed in the HJC model. When examining the same test data by plotting volumetric strain rather than axial strain, it is clear that only the CSC and KCC models are capable of modelling the volumetric expansion exhibited by the SAM-35 concrete near $f_{c}^{\prime}$. The HJC model continues to contract, while the BDC model remains linear.
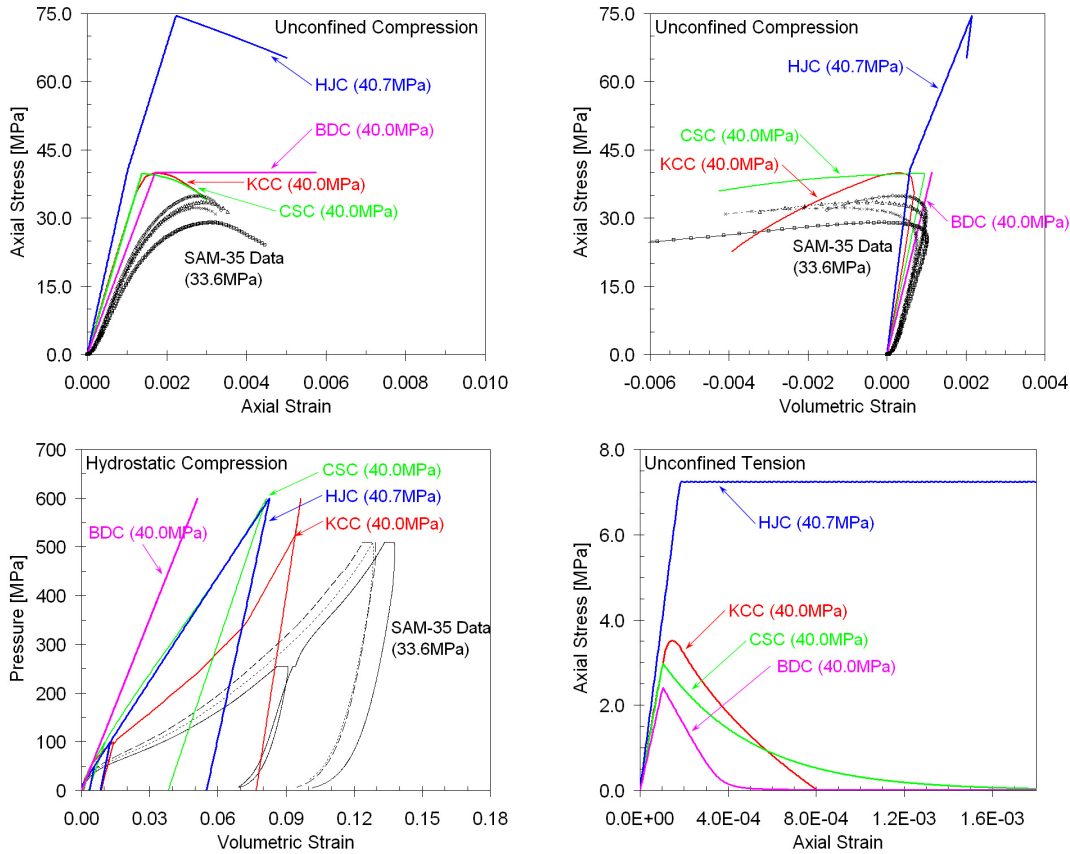

Figure 1: Comparison of models under basic stress paths.

The SAM-35 data also exhibits a trend common to most geo-materials under the hydrostatic compression stress path. This is noted in Figure 1 by an initial 
linear (or nearly linear) regime, a non-linear compaction region, and a volumetric lockup region. The $\mathrm{HJC}, \mathrm{CSC}$, and $\mathrm{KCC}$ models show this trend, although it is apparent that both the $\mathrm{HJC}$ and $\mathrm{CSC}$ models were calibrated to a different set of test data than was used for the KCC model. It is also obvious from Figure 1, that the BDC model uses a linear equation of state, which explains the linearity of the volumetric behaviour that is observed under the unconfined compression stress path. The HJC, CSC, and KCC models thus appear to possess the appropriate phenomenology, while the BDC model does not.

Figure 1 also shows comparisons between the various models under unconfined tension. Unfortunately, the SAM-35 test data only provided the peak tension during the tests $(0.8 \mathrm{MPa})$ and cannot be compared directly with the results from each model. Nevertheless, it is widely accepted that all conventional concretes exhibit a non-linearly decaying softening branch after fracturing in tension. All models capture this behaviour except the HJC, which predicts perfect plasticity and consequently over-predicts the energy absorbing capacity of concrete.

\subsubsection{Tri-axial stress paths}

Tri-axial stress path tests using various confinement pressures are representative of key loading conditions for RC structural components because they characterize the effects of confinement on concrete strength and ductility. Figure 2 shows results for single elements under tri-axial stress paths for various hydrostatic pressures. The SAM-35 data is plotted over the results from each of the models, in principal stress difference (PSD, axial stress minus radial stress, which is related to $\sigma_{e}$ ) versus axial strain space.

In these figures, the SAM-35 concrete exhibits an increase in strength under increasing confinement pressure. Another less appreciated phenomenon is evident from the test data, which is related to an increase in the material's ductility when confined. Note that at a $5 \mathrm{MPa}$ confining stress, the SAM-35 concrete appears similar to the unconfined compression results (albeit with a small strength enhancement). However, the SAM-35 exhibits a transition from brittle to ductile behaviour between 20 and $50 \mathrm{MPa}$ of confining stress. This is manifested by a transition from a loss of strength and a softening behaviour after achieving the maximum PSD for lower values of confinement, while for high values of confinement, hardening or perfectly plastic behaviour occurs. It is evident from the plots of Figure 2, that the models all capture the effects of confinement except for the BDC model that is clearly incapable of capturing the enhancements in strength and ductility. However, only the KCC model produces a believable pattern of failure envelopes.

\subsection{Comparison of models for structural response}

Although it is clear that there are some important phenomenological limitations in several of the models studied, it is difficult to ascertain the consequences this has on structural response calculations. To address this issue, two structural response problems were run to assess the performance of each constitutive model and its affect on the results produced. 

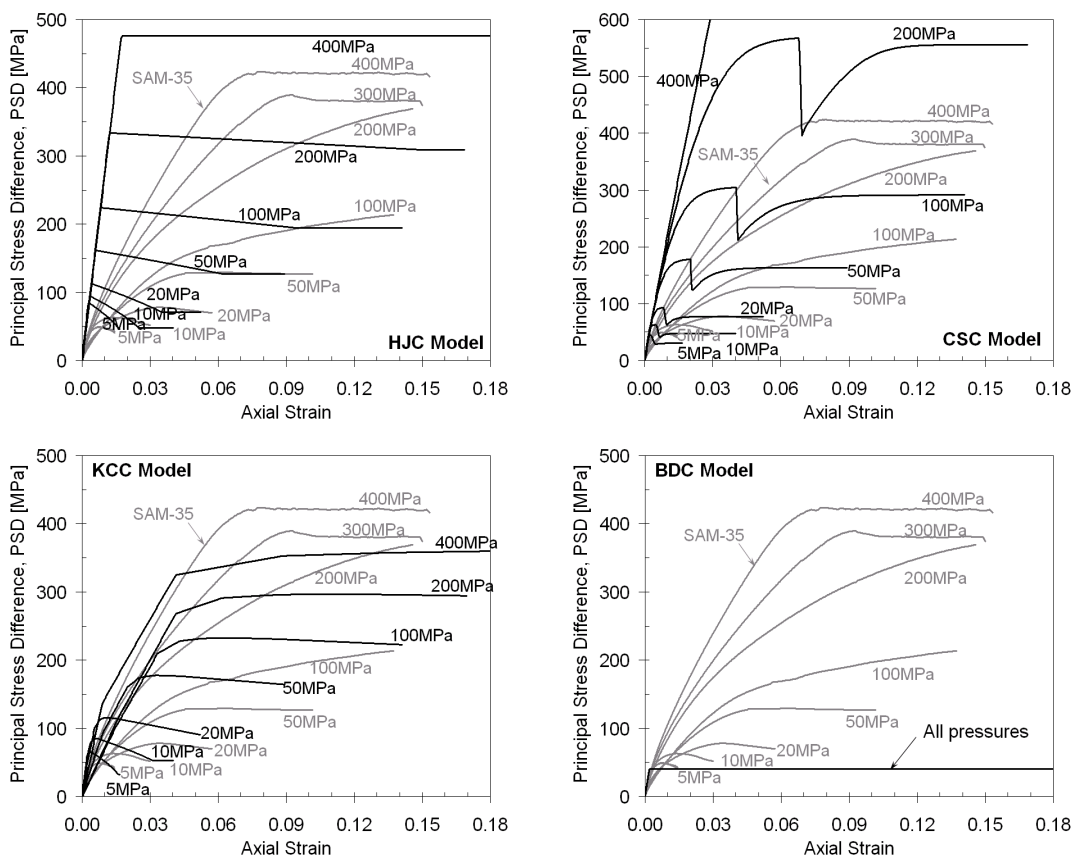

Figure 2: $\quad$ Comparison of models under tri-axial stress.

\subsubsection{RC column response}

The first problem involved the blast response of a column constructed with 45.0 MPa concrete and ASTM Grade 60 steel reinforcement. An FE model was developed for this $35.5 \mathrm{~cm}$ square RC column, which was subjected to the blast effects from a nearby bare high explosive charge [12]. Continuum elements were used for the concrete and beam elements used for the reinforcement. The pressure histories recorded during the actual test were applied to the FE model.

The actual column sustained a shear failure early in the response, leading to a large-deformation tensile membrane response. As a result, the column sustained $28 \mathrm{~cm}$ of displacement, but did not break free of the building as shown in Figure 3. This figure also shows the deformed shape computed by the four different FE models. The colour of the fringes shown on the deformed shape plots indicate the level of damage computed. The HJC and BDC models severely under-predict the column response. The CSC model, although clearly predicting local cracking over the column height, indicates an erroneous localized shear failure at approximately $1 / 4$ column height. The KCC model appears to represent the overall deformation of the test column fairly well. Figure 4a compares the displacement history at the column's mid-height for each calculation, and points to the inadequacy of the HJC and BDC models to predict the column response. No material erosion was allowed for these calculations. 


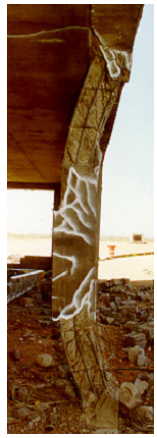

Test result

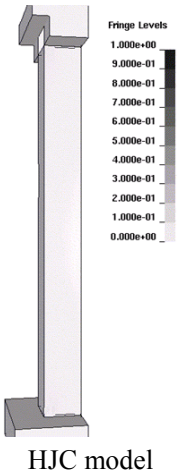

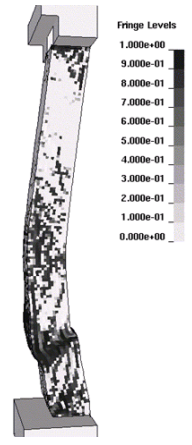

CSC model

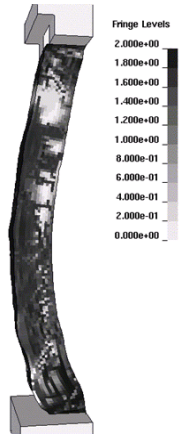

KCC model

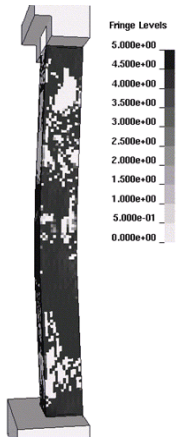

BDC model

Figure 3: $\quad$ Post-test deformation of the RC column.

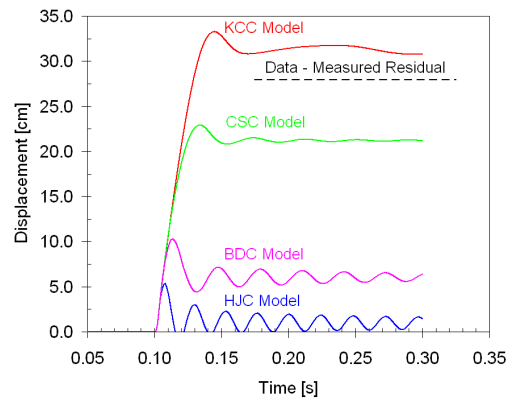

(a) Column

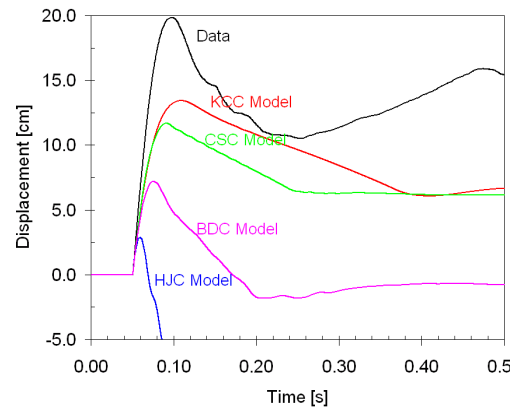

(b) Wall

Figure 4: Calculated RC column and wall displacements.

\subsubsection{RC wall response}

The second problem involved predicting the response for a $30 \mathrm{~cm}$ thick $\mathrm{RC}$ wall tested at the University of California at San Diego blast simulator [13]. The wall had a concrete compressive strength of $42.3 \mathrm{MPa}$, was lightly reinforced in both the vertical and horizontal directions with ASTM Grade 60 reinforcement, and was simply supported with a clear span of $330 \mathrm{~cm}$ [14]. The blast simulator uses an array of impact pads, which in this case struck the wall at $14.6 \mathrm{~m} / \mathrm{s}$, inducing an average impulse of 712 psi-ms. This load was characterized in the FE model by multiplying the measured impactor accelerometer data, multiplying by the impacting mass, and applying this force as pressure tractions on the wall.

The simulations yield results that are consistent with those of the preceding RC column problem. The HJC and BDC models under-predict the response of the wall, while the $\mathrm{CSC}$ and $\mathrm{KCC}$ models compare much more favourably. Figure 5 illustrates this with a post-test photograph of the wall and deformed shapes of each FE mesh at $75 \mathrm{~ms}$ into the wall response (including $50 \mathrm{~ms}$ of gravity body force initialization). Figure $4 \mathrm{~b}$ shows the wall mid-span displacement histories of each model with that recorded in the test. The wall had a peak displacement of $19.8 \mathrm{~cm}$, while the $\mathrm{KCC}$ model predicts $13.4 \mathrm{~cm}$, the 
CSC yields an $11.8 \mathrm{~cm}$ displacement, the BDC a $7.18 \mathrm{~cm}$, and the $\mathrm{HJC}$ predicts a $2.85 \mathrm{~cm}$ peak deformation.

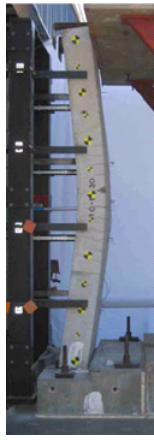

Test result

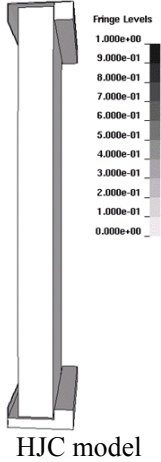

Figure 5:
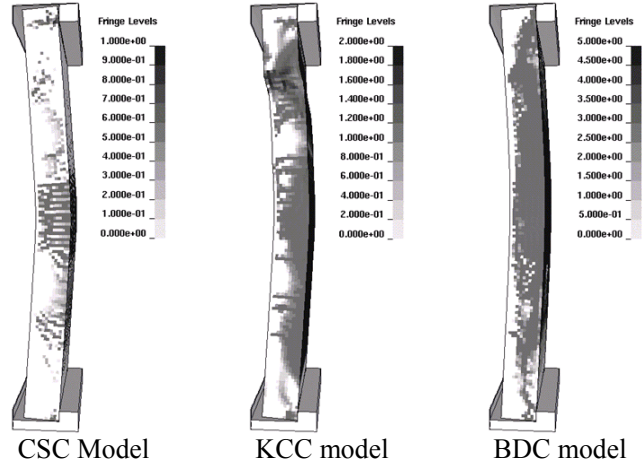

Post-test deformation of the RC wall.

\section{Concluding remarks}

The results of the single element stress path and structural response studies presented here point out several important limitations in several widely available concrete constitutive models in the LS-DYNA code. The HJC and BDC models were found to be largely inadequate to predicting structural response to impulsive loading. This is not surprising in the case of the one-invariant BDC model, but the HJC model exhibits a two-invariant failure surface formulation (a minimum for concrete) and has been validated in a number of penetration analyses. However, the results of these studies indicate that the HJC model, as currently implemented in LS-DYNA, does not adequately characterize concrete fracture in tension or the dilatancy effects under shearing. The KCC and CSC models, which performed relatively well in the structural response calculations, were shown to adequately capture both of these features. This is despite the finding that the CSC model showed some anomalies during compression softening.

In all fairness though, the constitutive model fits used in this study were calibrated to different concrete materials, which were then compared to completely different concretes of similar strengths. This makes it difficult to draw any directly quantitative conclusions from these studies, although the studies do point to the ability of the models to represent a generic concrete in various structural applications. In addition, two important issues are emphasized by the results of this study. First, this study illustrates the importance of conducting basic single element stress path investigations; the results of those presented here provided insight into the adequacy of each model to predict structural behaviour and should be performed by an analyst, novice or expert, prior to employing any of these models in structural response problems. What was shown here is this regard should be taken as a basic minimum; other important stress and strain paths were not shown and can be important depending on the class of the structural response problem. Secondly, validation with a 
variety of large scale analysis should be completed to insure that the overall behaviour prediction is qualitatively acceptable, even if the exact parameter fit or material characterization is not available. Once these checks are completed, better material characterization and parameter fits can be addressed.

\section{References}

[1] Livermore Software Technology Corporation, LS-DYNA user's manual Version 971, Livermore, CA, May, 2007.

[2] Holmquist, T.J., Johnson, G.R., and W.H. Cook, A computational constitutive codel for concrete subjected to large strains, high strain rates, and high pressures, Proceeding of the 14th International Conference on Ballistics, Quebec, Canada, pp. 591-600, 1993.

[3] Schwer, L.E., and Y.D. Murray, A three-invariant smooth cap model with mixed hardening, International Journal for Numerical and Analytic Methods in Geomechanics, Vol. 18, pp. 657-688, 1994.

[4] Malvar, L.J., J.E. Crawford, J.E. Wesevich, and D. Simons, A plasticity concrete model for DYNA3D, International Journal of Impact Engineering, Vol. 19, Nos. 9-10, pp. 847-873, 1997.

[5] Govindjee, S., Kay, G.J., and J.C. Simo, Anistropic modeling and numerical simulation of brittle damage in concrete, International Journal for Numerical Methods in Engineering, Vol. 38, pp. 3611-3633, 1995.

[6] Sandler, I.S., DiMaggio, F.L., and G.Y. Baladi, Generalized cap model for geological materials, ASCE Journal of the Geotechnical Division, Volume 102, GT7, pp. 683-699, 1976.

[7] Rubin, M., A simple, convenient isotropic failure surface, ASCE Journal Engineering Mechanics, Volume 117, No. 2, pp. 348-369, 1991.

[8] Chen, W.F. Plasticity in reinforced concrete, McGraw-Hill: NY, 1982.

[9] Williams, E.M., Akers, S.A., and P.A. Reed, Laboratory characterization of SAM-35 concrete, US Army Corp of Engineers Engineering Research and Development Center Technical Report, TR-06-15, September, 2006.

[10] Thacker, B.H., Probabilistic Sensitivity Analysis of the HolmquistJohnson-Cook Material Model for Concrete, Briefing presented to the Air Force Research Laboratory, August, 2000.

[11] Malvar, L.J., Crawford, J.E., and K.B. Morrill, K\&C concrete material model release III - automated generation of material model input, Karagozian \& Case Technical Report, TR-99-24.1, August, 1999.

[12] Malvar, L.J., Morrill, K.B., and J.E. Crawford, CTS-1 retrofit designs for reducing the vulnerability of an office building to air blast, Karagozian \& Case Technical Report, TR-98-39.2, Burbank, CA, September, 1998.

[13] Gram, M.M., Clark, A.J., Hegemier, G.A., and F. Seible, Laboratory simulations of blast loading on building and bridge structures, Structures Under Shock and Impact IX, eds. N. Jones, WIT Press: Southampton, pp. 33-44, 2006.

[14] Seible, F., Hegemier, G.A., and M. Oesterle, DYNABLOK wall test series dynamic test 5 summary RC-D-30, University of California San Diego Test Report, N41756-05-C-4762, August, 2006. 